\title{
Fatigue investigation of complex weldments by the means of the local strain energy density approach
}

\author{
Luigi Mario Viespoli ${ }^{1}$, Antonio Alvaro ${ }^{2},{\text { Bård } \text { Nyhus }^{2} \text {, and Filippo Berto }}^{1}$ \\ ${ }^{1}$ Department of Mechanical and Industrial Engineering, Norwegian University of Science and Technology (NTNU), Norway \\ ${ }^{2}$ Department of Materials and Nanotechnology, SINTEF Industry, Norway
}

\begin{abstract}
The paper investigates the use of an energetic approach based on the strain energy density failure criteria to predict the fatigue life of welded joints in aluminum alloy. The cited criterion has already been proved valid to assess the failure of components in presence of sharp and blunt notches, and several results are present in the literature for different materials. The geometry tested in the present work is a double V-grooved full penetration butt weld, subsequently heat treated, and loaded orthogonally to the welding direction with load ratio $\mathrm{R}=0$. This configuration makes the weld toe a notch of great opening angle. The aim of the paper is to verify the soundness of the energetic criteria for this class of welded joints, comparing the tests results with the numerical predictions. In the computational part, the energy in a given volume can be computed directly from the nodal displacements, thus not needing the stress values. Differently from the stress intensity factor approach, this property allows to a fast computation of the strain energy density by the means of a coarse mesh. The results of different configurations of geometry and meshing are compared to find the simplest modeling scheme capable of providing an accurate estimate of the fatigue life of the joint.
\end{abstract}

\section{Introduction}

The fatigue life assessment of welded components can be executed with different techniques [1] of which, due to their great simplicity, commonly used are the Nominal Stress and the Hot Spot Stress approaches [2]. The drawback of this simplicity, which makes them ideal in complex structures with a great number of welds, the detail they provide regarding the stress field in the stress intensification area is poor, so they are strongly related to the determination of the right fatigue class for every welded detail. In the presence of a sharp notch, that is a weld toe or root, the static and fatigue resistance can be evaluated using the Notch Stress Intensity Factors [3]. Such procedure presents two drawbacks: the necessity of an accurate evaluation of the stress field [4], so a very refined discretization and a non-constant critical value, but function of the opening angle through the William's eigenvalues [5]. The use of special elements [6], capable of describing the asymptotic behavior in proximity of the stress singularity point, can be helpful in the reduction of the number of elements, but often this is not implemented in commercial codes. The adoption of the mean Strain Energy Density for the fatigue life prediction [7] allows to a rapid and accurate assessment and to the computation a-posteriori of the Notch Stress Intensity Factors [8] with a closed-form analytical correlation. Since the SED is computed directly from the stiffness matrix and nodal displacement, without the need of the stress solution, its value is accurate also for very coarse meshes [9], providing an evident computational advantage. The local energetic criterion states that the failure occurs when the mean SED, $W$, averaged over a control volume of precise radius $R$, equals a critical value $W_{c}$, not dependent on the geometrical characteristics of the notch, but only on the material properties, as it is the radius $R$. Dealing with a sharp V-notch and considering a bi-dimensional problem, the energy is computed over a circular area $\Omega$ of radius $R$ which center is the notch tip and symmetric with respects to the notch bisector. In the hypothesis of validity of the William's solution, the mean SED over the critical area is:

$$
\bar{W}=\frac{e_{1}}{E}\left[\frac{K_{1}}{R^{1-\lambda_{1}}}\right]^{2}+\frac{e_{2}}{E}\left[\frac{K_{2}}{R^{1-\lambda_{2}}}\right]^{2}
$$

Being $\lambda_{1}$ and $\lambda_{2}$ the William's eigenvalues, $E$ the Young's modulus and $e_{1}$ and $e_{2}$ functions of the notch's opening angle and of the stress state. The Notch Stress 
Intensity Factors $K_{I}$ and $K_{2}$ can be computed from the stress field as:

$$
\begin{aligned}
& K_{1}=\sqrt{2 \pi} \lim _{r \rightarrow 0}\left(\sigma_{\theta}\right)_{\theta=0} r^{1-\lambda_{1}} \\
& K_{2}=\sqrt{2 \pi} \lim _{r \rightarrow 0}\left(\tau_{r \theta}\right)_{\theta=0} r^{1-\lambda_{2}}
\end{aligned}
$$

The fatigue life assessment by the means of the SED has been validated for welded joints and both sharp and blunt notches $[10,11]$. If the opening angle is greater than $103^{\circ}$, the Mode II stress field is not singular, so its contribution to the strain energy around the notch tip is negligible compared to the contribution of the Mode I, allowing to express the mean SED as:

$$
\bar{W}=\frac{e_{1}}{E}\left[\frac{K_{1}}{R^{1-\lambda_{1}}}\right]^{2}
$$

An estimate of the radius $R$ of the control area $\Omega$ can be estimated in the hypothesis of validity of the Beltrami failure criterion, $W_{c}=\Delta \sigma / 2 E$, and applying it to tests on butt welded joints. The critical SED so found is then compared to the mean SED in the case of a welded joint with opening angle greater than $103^{\circ}$, obtaining:

$$
R=\left(\frac{\sqrt{2 e_{1}} \Delta K_{1}}{\Delta \sigma}\right)^{\frac{1}{1-\lambda_{1}}}
$$

It is important to point out the dependence of the critical radius to the failure criterion applied. In the case of aluminum welded joints, the radius found with a number of experimental tests is $R=0.12 \mathrm{~mm}$ [12].

\section{Joint fatigue testing}

\subsection{Specimens}

The main goal of the fatigue testing of the welded samples is to evaluate the fatigue performance of double $\mathrm{V}$-groove butt welds of heat-treated aluminum alloy at room and low temperature. The specimens have been manufactured joining two AA6082-T6 plates with a MIG welding process and then cutting and machining the single specimens to a width of $30 \mathrm{~mm}$. The shielding gas used is argon and two series of samples have been tested: 5 and $20 \mathrm{~mm}$ of thickness. The size of the plates was originally $180 \times 20 \times 2000 \mathrm{~mm}$ and $150 \times 5 \times 2000 \mathrm{~mm}$. No post welding treatment has been performed.

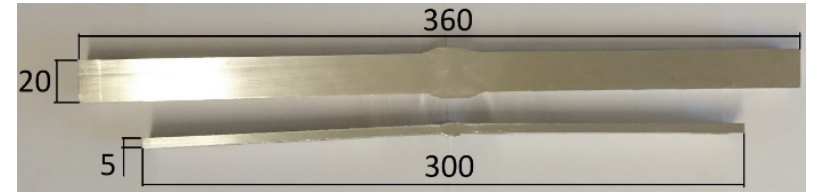

Figure 1. Two of the tested specimens, all cut to a width of $30 \mathrm{~mm}$. It is evident the pronounced bending induced by the welding process, especially for the $5 \mathrm{~mm}$ thick sample.

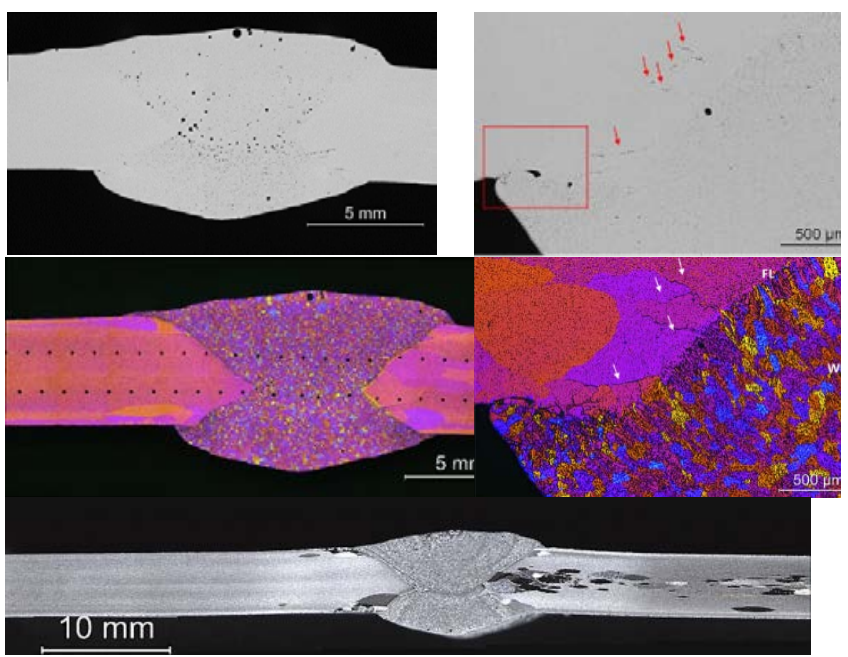

Figure 2. A metallographic investigation of the junction, in the pictures for the $5 \mathrm{~mm}$ thick joints, clearly shows

the presence of defects such as porosity in the penetration zone, cracks in the HAZ (arrows) and a root defect (frame). The base material can be observed to have partly recrystallized.
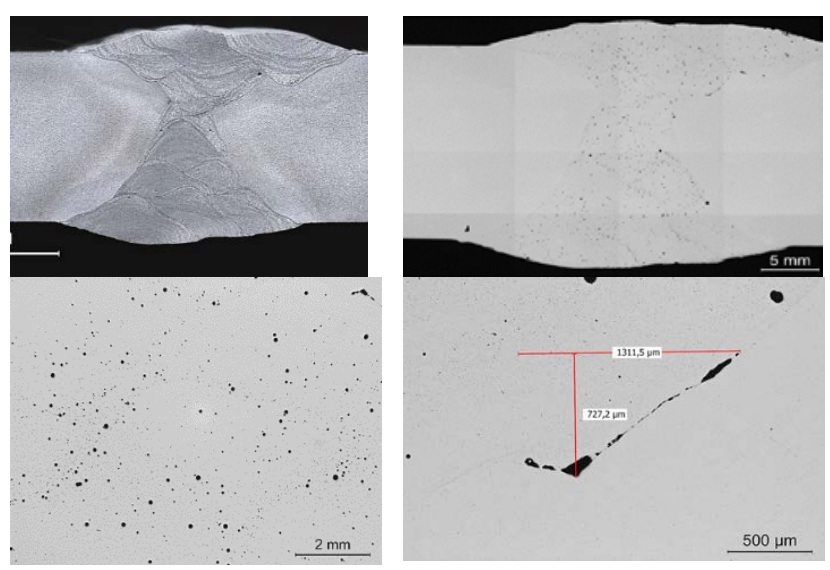

Figure 3. As in figure 2, also the $20 \mathrm{~mm}$ joints present porosity and cracks.

After production, the samples present a noticeable angular misalignment, particularly pronounced in the case of the $5 \mathrm{~mm}$ joints. This geometrical characteristic, due to the rigid clamping system, heavily influences the effective load acting on the specimen. This effect is taken into account in the computation according to the recommendations of IIW, as explained in the following chapter.

\subsection{Testing and fatigue curves}

The fatigue texting has been executed at a load ratio $\mathrm{R}=0$ in air with a sinusoidal load cycle, at room temperature and at $-60{ }^{\circ} \mathrm{C}$. The equipment used is a MTS servohydraulic machine with a load capacity of $100 \mathrm{kN}$. The nominal stress increment due to the angular misalignment between flat plates, in the case of fixed ends, is obtained multiplying the nominal longitudinal stress imposed by the machine times a coefficient $k_{m}$, 
function of the geometrical and elastic characteristics of the joint.

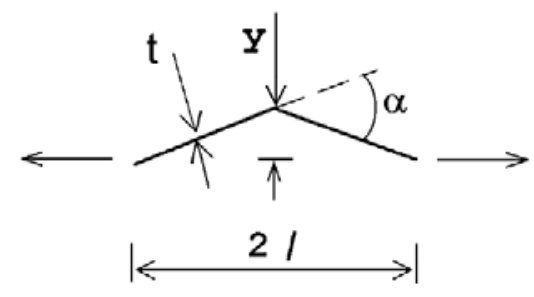

$$
k_{m}=1+\frac{3 y}{t} \cdot \frac{\tan (\beta / 2)}{\beta / 2}
$$

With:

$$
\beta=\frac{2 l}{t} \sqrt{\frac{3 \sigma_{m}}{E}}
$$

Figure 4. Corrective factor for angular misalignment according to IIW.

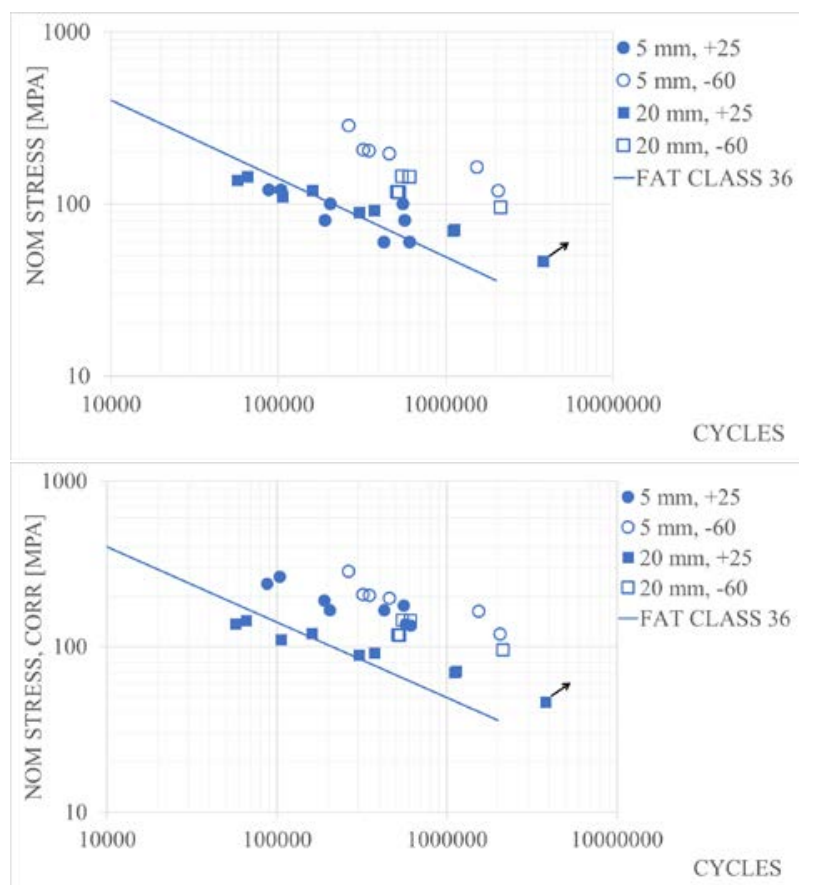

Figure 5. Confront between the fatigue curve of the FAT CLASS 36, the resistance proposed by IIW for V-groove non-grounded butt welds in aluminum alloy. The results

are reported both for the non-corrected (top) and the corrected (bottom) nominal traction stress. The run out is marked by an arrow.

The results obtained in terms of fatigue life are in accordance with the minimum duration obtained by the fatigue prediction with nominal stress and the correct fatigue class stress. It is evident, by the comparison of the non-corrected and the corrected results, the importance of taking into account the distortion of the joints due to the welding process. From the corrected plot, Figure 5 left, two effects are evident: an increase in fatigue life for the tests at low temperature and a better behavior of the joints realized with thinner plates. This thickness effect, explained in paragraph 4 , is due to the increase of SED in the critical volume associated to an increase in thickness for two geometrically similar joints.

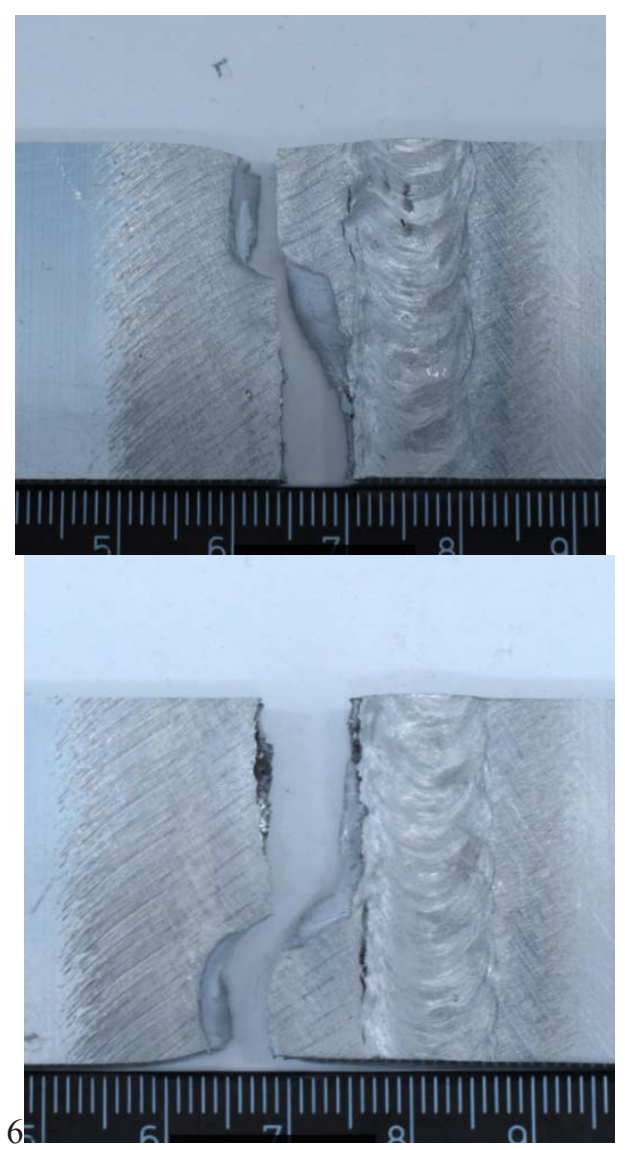

Figure 6. Crack initiation points (hot spots) for the $5 \mathrm{~mm}$ thick plate at room (top) and low temperature (bottom). The fatigue crack initiates at the stress intensification point and follows approximately the Heat Affected Zone, with final plastic fracture, in the case of both testing temperatures.

\section{SED analysis}

\subsection{FEM modeling}

The local mean strain energy density has been proved to be a valid and reliable tool of fatigue prediction in the presence of stress intensification due to the geometry of the component [4]. The main objective of this paper is to verify whether this tool can be applied to the case of a butt-weld joint, in which the notch opening angle and the weld size have a high degree of variability. The property of the SED of being independent of the notch opening angle plays a key role for the fitness of the method to be applied to this problem. For the finite element analysis the joint is modeled as an ideal linear elastic continuum, thus neglecting the material's defects and residual stresses after the welding process. In fact, in a similar way to what is done for the fatigue assessment with 
nominal stress and fatigue class, these effects are already considered in the fatigue band of the material. Regarding modelling the geometry, the real dimensions of the joint, particularly its deflection and width and height of the weld have been measured and averaged. The weld has been modeled then as an ideal arc passing through the two weld toes and the center point of the weld. Assuming symmetrical geometry, only a quarter of the joint has been modeled and it has been subject to a unit traction load. The distortion/misalignment is not taken into account in the model, which therefore simulate a straight joint, but is used to compute the corrected stress from the nominal stress according to IIW. The mean SED at the weld toe for a generic nominal load is then easily computed by multiplying the mean SED of the unit load times the square of the nominal load applied. For this type of joint, the geometrical weld toe stress concentrator induce crack initiation. To predict the crack initiation life, approximated to the total life of the specimen, the critical volume of radius $\mathrm{R}=0.12 \mathrm{~mm}$ is centered in the weld toe. The very low sensibility of the mean SED to the mesh refinement allows to the use of a coarse discretization, while the stress state has been set to plain strain. The load application edge in the model is set $50 \mathrm{~mm}$ distant from the edge of the specimen, according to the clamping distance.
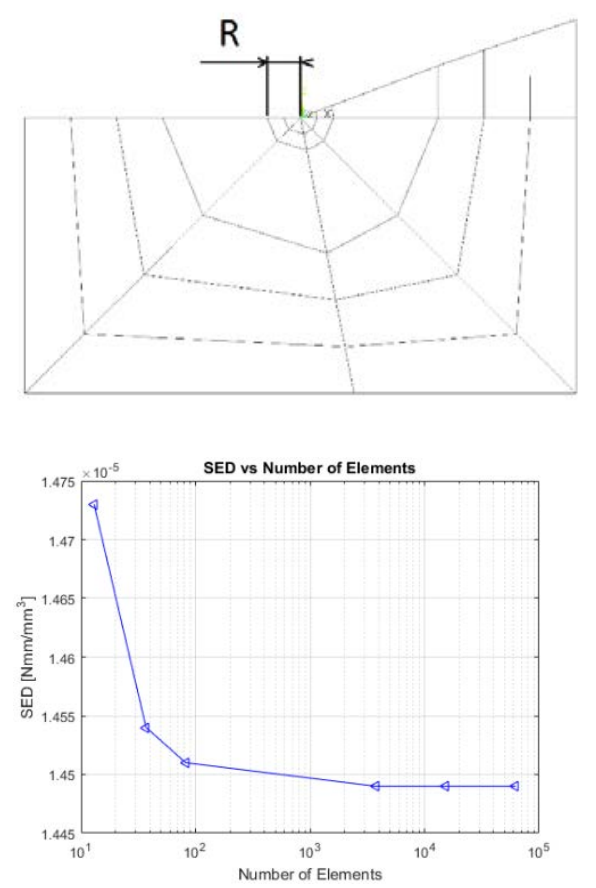

Figure 7. Coarse mesh around the weld toe and critical volume of radius $\mathrm{R}=0.12 \mathrm{~mm}$ (top). Mean SED for unit traction stress as a function of the number of elements: it is evident how a fine mesh is not necessary to the local energetic approach (bottom). Both figures refer to the 5 $\mathrm{mm}$ thick plates.

\subsection{SED fatigue band}

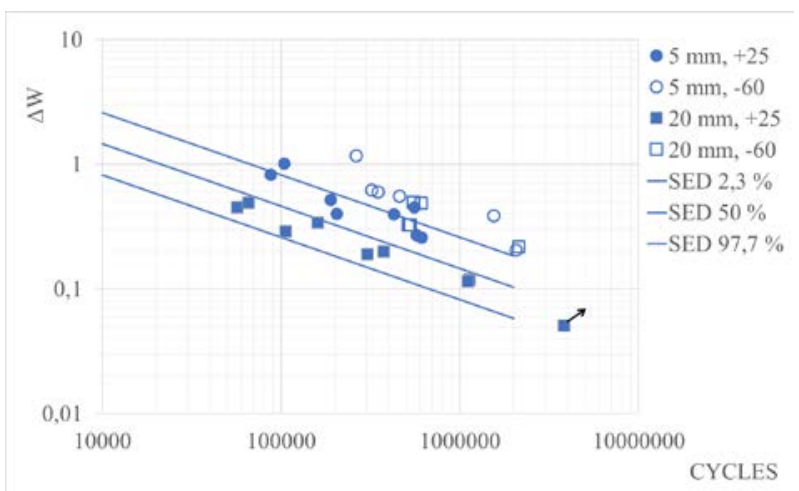

Figure 8. Summary of the results obtained in terms of mean strain energy density computed from the corrected stress.

Comparing the summary of the results obtained in terms of mean SED computed from the corrected stress to the results in terms of nominal stress the scatter is decreased. The results at room temperature are concentrated in the fatigue band available in the literature for welded aluminum joints, with a higher resistance for the $5 \mathrm{~mm}$ thick plates. A strong increase in fatigue life is observed for the specimens tested at low temperature, due to the slower crack initiation and propagation.

\section{Thickness effect}

From the analysis of the results, it is clear an influence of the thickness of the joint on the total fatigue life, with a better performance for thinner plates. Even though this is not reported for the detail tested in the IIW document, the effect has important impact on the joint's behavior and can be easily explained recurring to an analytical description based on the theory of the Strain Energy Density [3]. Being "a" and "b" two geometrically similar joints, of characteristic dimensions $t_{a}$ and $t_{b}$, being " $i$ " 1 or 2 according to the loading mode and being $\lambda_{\mathrm{i}}$ the corresponding William's eigenvalue, the N-SIFs of the two joints are correlated by:

$$
K_{i, b}=K_{i, a}\left(\frac{t_{b}}{t_{a}}\right)^{1-\lambda_{i}}
$$

The mean SED can be re-written in terms of N-SIFs and geometrical parameters:

$$
\Delta \bar{W}=\frac{1}{E} e_{1} K_{1}^{2} R^{2\left(\lambda_{1}-1\right)}+\frac{1}{E} e_{2} K_{2}^{2} R^{2\left(\lambda_{2}-1\right)}
$$

If the notch opening angle is higher than $102^{\circ}$, only the Mode I loading is singular and the Mode II is negligible. The mean SED for the two joints is expressed as: 


$$
\left\{\begin{array}{l}
\Delta \bar{W}_{a}=\frac{1}{E} e_{1} K_{1, a}^{2} R^{2\left(\lambda_{1}-1\right)} \\
\Delta \bar{W}_{b}=\frac{1}{E} e_{1} K_{1, b}^{2} R^{2\left(\lambda_{1}-1\right)}
\end{array}\right.
$$

Considering the thickness of the samples tested, the correlation between the mean SED for the two types is:

$$
\begin{gathered}
\Delta \bar{W}_{b}=\Delta \bar{W}_{a}\left(\frac{t_{b}}{t_{a}}\right)^{2\left(1-\lambda_{1}\right)}=\Delta \bar{W}_{a}\left(\frac{20}{10}\right)^{2(1-0.674)} \\
=\Delta \bar{W}_{a} \cdot 157
\end{gathered}
$$

Considering the result of the FEM analysis, the mean SED for a unit traction stress at the weld toe for the 20 $\mathrm{mm}$ and the $5 \mathrm{~mm}$ joints, are $2.38 \mathrm{E}-5$ and $1.45 \mathrm{E}-5$ respectively, in a ratio of 1.64 , close to the value found by the analytical approximation. This explains how two similar joints of different size can have a significantly different fatigue life when subjected to the same nominal stress.

\section{Conclusions}

In the present paper, the results of a series of longitudinal traction fatigue tests performed on two different thicknesses, 5 and $20 \mathrm{~mm}$, butt weld aluminum joints are presented. The tests have been executed both at room and at low temperature $\left(-60{ }^{\circ} \mathrm{C}\right)$, in as welded condition. The main goals of the study are two: understanding the effect of size, dimensional deviations and temperature on the fatigue life and verifying the fitness of the application of the local energetic method of the mean SED to the rapid fatigue assessment for buttwelded joints. Three main conclusions are drawn from the tests executed. The mean SED approach is suitable to predict the fatigue behavior for $\mathrm{V}$-groove butt welds, with a simple 2D model based on the average size and distortion of the joints, providing more accurate results than the Nominal Stress approach. For the alloy and the welding technology object of the study, the thicker plates $(20 \mathrm{~mm})$ show a reduced fatigue life compared to the thinner ones $(5 \mathrm{~mm})$, how explained in paragraph 4 . Moreover, the testing at low temperature shows an important increase in fatigue life compared to the results at room temperature. The average increase in fatigue life at low temperature is of 3 and 6 times for the $5 \mathrm{~mm}$ and $20 \mathrm{~mm}$ thick joints respectively, but the number of samples tested so far doesn't allow to a safe and certain numerical quantification of the effect.

The authors wish to thank the Research Council of Norway for funding through the Petromaks 2 Programme, Contract No.228513/E30. The financial support from ENI, Statoil, Lundin, Total, Scana Steel Stavanger, JFE Steel Corporation, Posco, Kobe Steel, SSAB, Bredero Shaw, Borealis, Trelleborg, Nexans, Aker Solutions, Marine Aluminum, FMC Kongsberg Subsea, Hydro and Sapa are also acknowledged.

\section{References}

[1] Eurocode 3: Design of steel structures - Part 1-9: Fatigue, EUROPEAN COMMITTEE FOR STANDARDIZATION Ref, No, EN 1993-1-92005: E

[2] Hobbacher A., RECOMMENDATIONS FOR FATIGUE DESIGN OF WELDED JOINTS AND COMPONENTS IIW document IIW-1823-07

[3] Lazzarin P, Tovo R. A notch intensity factor approach to the stress analysis of welds. Fatigue \& Fracture of Engineering Materials \& Structures 1998; 21: 1089-1103.

[4] Lazzarin P, Zambardi R. A finite-volume-energy based approach to predict the static and fatigue behavior of components with sharp V-shaped notches. International Journal of Fracture 112: 275-298, 2001.

[5] Williams ML. Stress singularities resulting from various boundary conditions in angular corners of plates in tension. $\mathrm{J}$ Appl Mech 1952;19:526-8.

[6] Henshell RD, Shaw KG. Crack tip finite elements are unnecessary. Int J Numer Methods Eng 1975; 9:495-507

[7] Filippo Berto, Paolo Lazzarin, The volume-based Strain Energy Density approach applied to static and fatigue strength assessments of notched and welded structures, Procedia Engineering 01 (2009) 155-158

[8] Lazzarin P, Berto F, Gomez F J, Zappalorto M. Some advantages derived from the use of the strain energy densityover a control volume in fatigue strength assessments of welded joints. International Journal of Fatigue 30 (2008) 13451357.

[9] Lazzarin P, Berto F, Zappalorto M. Rapid calculations of notch stress intensity factors based on averaged strain energy density from coarse meshes: Theoretical bases and applications. International Journal of Fatigue 32 (2010) 15591567.

[10] Lazzarin, P., Berto, F., 2005. Some expressions for the strain energy in a finite volume surrounding the root of blunt V-notches. International Journal of Fracture, 135(1-4), 161185.

[11] Lazzarin, P., Berto, F., Elices, M., Gómez, J., 2009. Brittle failures from U- and V-notches in mode I and mixed, I + II, mode: A synthesis based on the strain energy density averaged on finite-size volumes. Fatigue \& Fracture of Engineering Materials \& Structures, 32(8), 671-684.

[12] Livieri, P. \& Lazzarin, P. Int J Fract (2005) 133: 247. https://doi.org/10.1007/s10704-005-4043-3 